\title{
Brick plots: an intuitive platform for visualizing multiparametric immunophenotyped cell clusters
}

Samuel E. Norton', Julia K. H. Leman', Tiffany Khong ${ }^{2,3}$, Andrew Spencer ${ }^{2,3}$, Barbara Fazekas de St Groth ${ }^{4,5}$, Helen M. McGuire ${ }^{4,5^{*}}$ and Roslyn A. Kemp ${ }^{1 *}$ (D)

* Correspondence: helen.mcguire@ sydney.edu.au; roslyn.kemp@otago. ac.nz

${ }^{4}$ Ramaciotti Facility for Human Systems Biology, The University of Sydney and Centenary Institute, Sydney, Australia

${ }^{1}$ Department of Microbiology and Immunology, University of Otago, Dunedin, New Zealand

Full list of author information is available at the end of the article

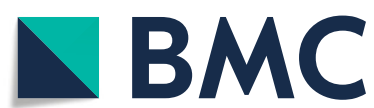

(c) The Author(s). 2020 Open Access This article is licensed under a Creative Commons Attribution 4.0 International License, which permits use, sharing, adaptation, distribution and reproduction in any medium or format, as long as you give appropriate credit to the original author(s) and the source, provide a link to the Creative Commons licence, and indicate if changes were made. The images or other third party material in this article are included in the article's Creative Commons licence, unless indicated otherwise in a credit line to the material. If material is not included in the article's Creative Commons licence and your intended use is not permitted by statutory regulation or exceeds the permitted use, you will need to obtain permission directly from the copyright holder. To view a copy of this licence, visit http://creativecommons.org/licenses/by/4.0/. The Creative Commons Public Domain Dedication waiver (http://creativecommons.org/publicdomain/zero/1.0/) applies to the data made available in this article, unless otherwise stated in a credit line to the data. 
does not use advanced analysis methods. A range of platforms and analysis tools that allow all immunologists and non-immunologists to use and understand these datasets are now available $[1,3,4,6,7]$. However, in our own prior research, the biggest hurdle to understanding and presenting the results of such analyses was the visualization of cluster phenotypes and the ability to clearly communicate results to a broad research audience.

Here we propose a new method for visualizing immune cell phenotypes downstream of cluster analyses. We generate a two-dimensional barcode that describes the phenotype of a given immune cell population. This barcode is called a Brick plot and comprises "bricks" that each represent a marker, with the position of the brick in twodimensional space reflecting its correlation to all other markers. We believe these Brick plots represent an intuitive approach to visualizing complex immune cell populations.

\section{Results}

\section{A two-dimensional barcode of cluster phenotype}

The principal aim of many high parameter analyses is to reduce complex data to a form that is readily interpretable by the analyst. This can be achieved with the use of clustering and dimensionality reduction techniques. Figure 1 provides a summary of five commonly used methods for visualizing mass cytometry data, together with four approaches used to illustrate the phenotype of clusters/populations defined by these methods. However, these four approaches are not always easily interpretable. Furthermore, these approaches often require considerable time and effort on the part of the researcher. We aimed to produce a visualization technique that resulted in a rapidly interpretable phenotypic readout for each cluster, regardless of the source of the cluster.

We propose a two-dimensional barcode for each cluster phenotype composed of bricks that represent each marker expressed in that cluster. We have termed these "Brick plots" (Fig. 2). For each defined cell cluster, bricks are located based on the coexpression correlation between their respective markers - bricks that represent markers that are commonly co-expressed in the given dataset are closely positioned, for example CTLA-4 and GATA3 in Fig. 2b Cluster B (top right). The relative size of each brick indicates the relative expression of that marker in that cluster compared to other clusters - a large brick indicates high relative expression and no brick indicates no, or low, relative expression. The result is an immediately interpretable visualization of the cluster phenotype (Fig. 2).

The Brick plot code can be found at https://github.com/NortonS1/BrickPlots. Users of this package are expected to have familiarity of how to install packages in R, although instructions on how to do this are included. The first step is to designate marker-brick position based on co-expression. A correlation matrix is produced using median marker expression within each cluster. This provides a value from -1 to 1 indicating the relative co-expression of each marker to each other marker within the dataset (Fig. 2a.1). Next a principal component analysis (PCA) is performed on the correlation matrix. Demarking the greatest variance as scalar projections, principal component (PC)1 and $\mathrm{PC} 2$ are used as the axes for determining brick-marker location (Fig. 2a.2). In addition to the PCA, an iterative K-means clustering step is performed to 


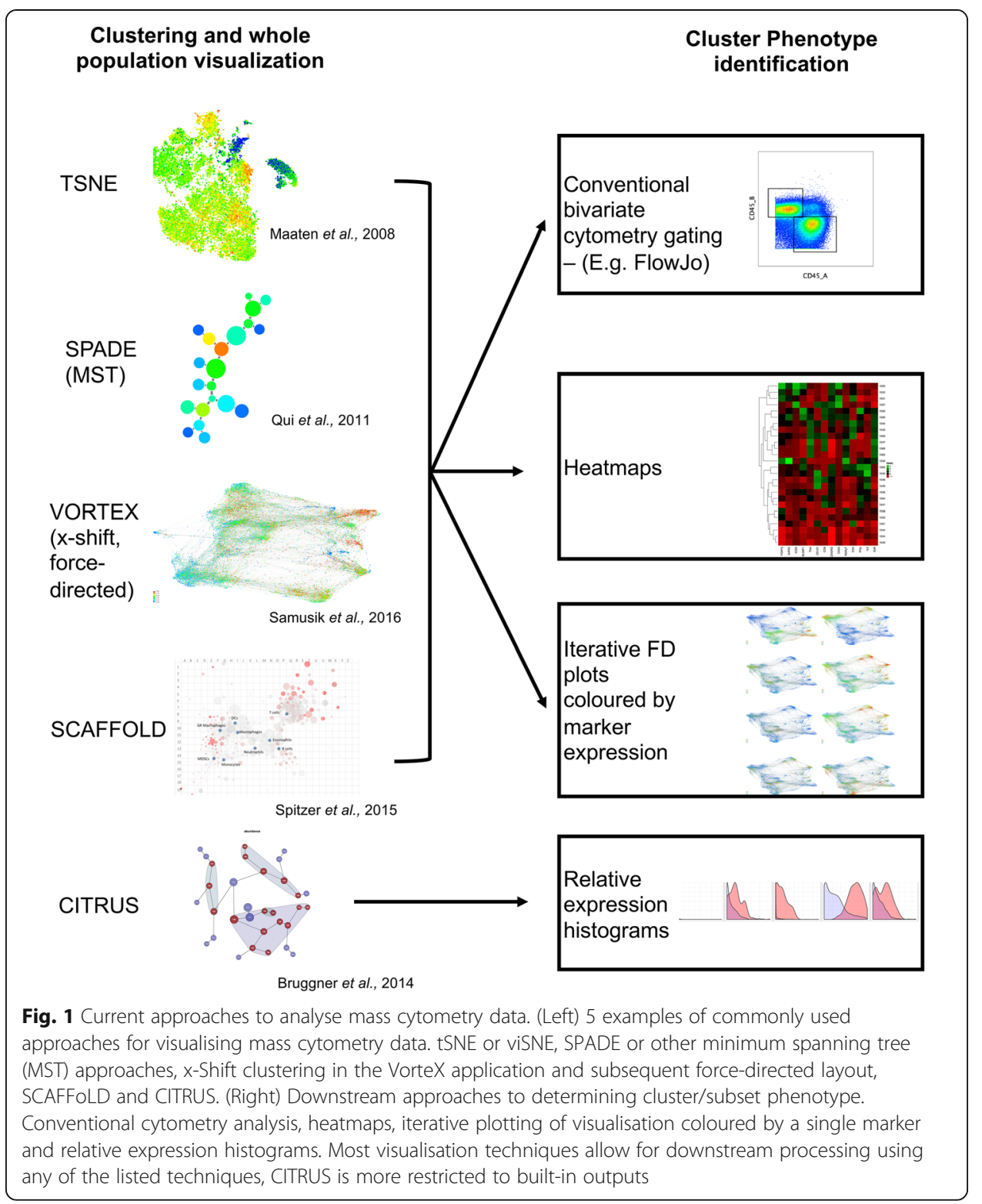

group the clusters. This is performed with $30 \mathrm{~K}$ values from one to the number of parameters in the dataset. Elbow point validation is used to decide the most accurate $\mathrm{K}$ value (tested between number1 and number2). Marker-brick positions are then grouped (by color) based on K-means group assignation (Fig. 2a.2). This approach not only groups markers for ease of interpretation, but can also provide additional information regarding co-expression of functional markers.

The second step is to place bricks at the previously defined locations. For this, the area of each given brick is a scaled representation of the arcsinh transformed expression value for that given marker in that cluster. This results in large bricks for highly expressed markers and small bricks for low, but still positive expression. Markers within a cluster with arcsinh transformed values below one for that marker are zeroed - resulting in no brick, which reduces noise and improves interpretability (Fig. 2a.4, b). We selected a value of one based on background signal reaching a maximum of one in 


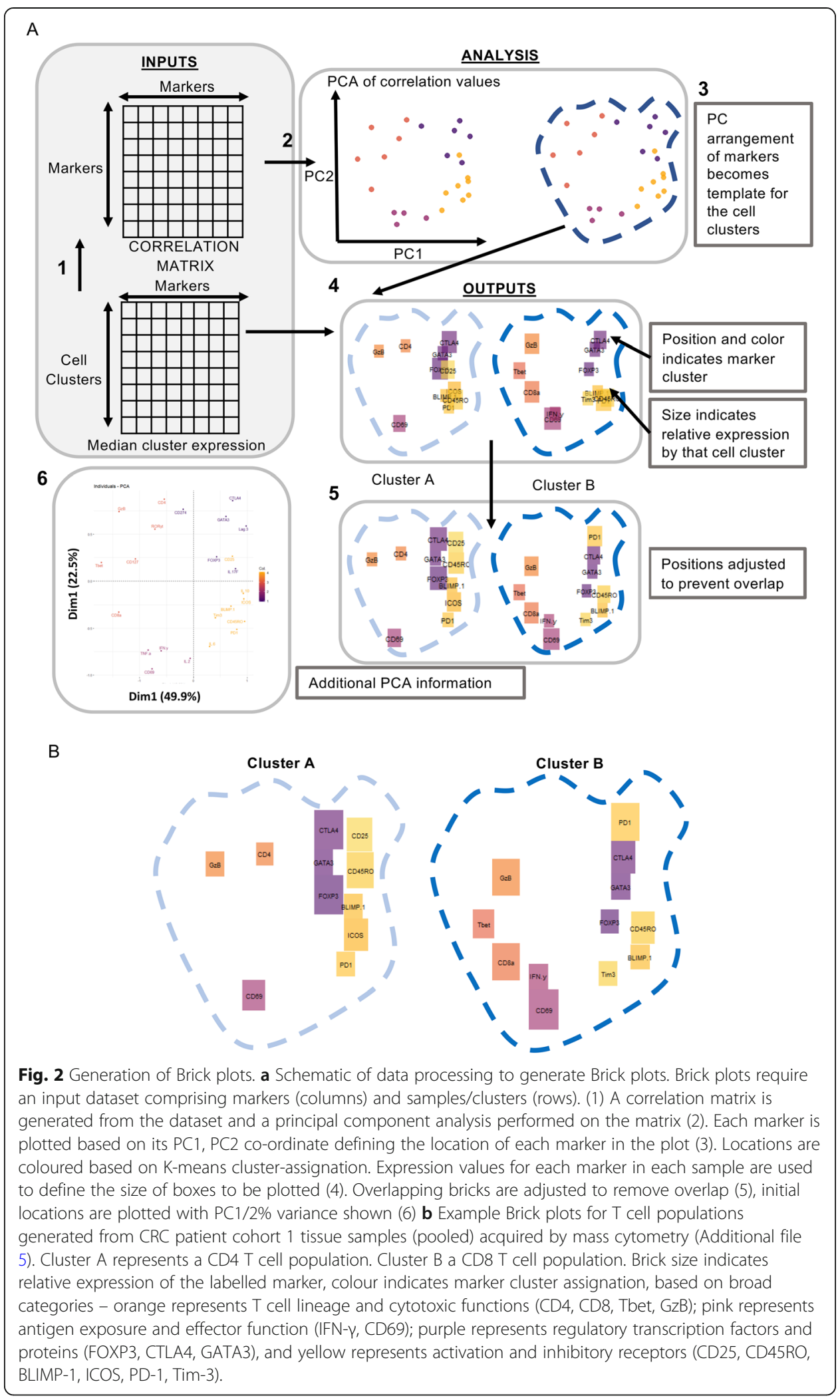


most markers, however, this value can be easily altered by the user to suit any given background threshold (Additional file 4). Arcsinh transformation is utilized as a convention with mass cytometry data, deemed suitable for transforming the measured ion counts as it resembles a log transformation in the upper range while retaining linearity for low counts. The range of the ion counts where linearity is retained can be adjusted by dividing counts by a user-defined factor before ArcSinh transformation, thus emphasizing the signal in the lower end of the spectrum. For CyTOF data, a co-factor around 5 is typically used-that is, all counts are divided by 5 to deemphasize noise around 0 .

This approach to brick localization often resulted in considerable brick overlap making interpretation of cluster phenotype challenging. As such, an additional step was added to detect for brick overlap and adjust the location by placing the new brick adjacent to the existing overlapped brick (Fig. 2a.5).

Figure $2 \mathrm{~b}$ depicts Brick plots for two clusters identified within mass cytometry data generated from Cohort 1 CRC tissue samples using VorteX. In Fig. 2b, cluster B has a large IFN- $\gamma$ brick (bottom left), indicating high relative IFN- $\gamma$ expression. The IFN- $\gamma$ brick is absent in cluster A indicating very low or no IFN- $\gamma$ expression. Cluster B, however, has some T-bet expression, although this expression is not as high as other populations in the analysis, indicated by a small brick.

Clusters A and B are a subset of the full complement of populations generated from Cohort 1 and shown in Additional file 1 as a heatmap, with a direct comparison to a single cluster expressed as a Minimum spanning tree (MST) plot or a Brick plot. Comparison of clusters A and B illustrated as Brick plots (Fig. 2b) with the heatmap (Additional file 1) revealed that all markers depicted in either of the two Brick plots (cluster 20,313 is A, cluster 20,315 is B) appear as green (high expression) boxes in the heatmap, and no red boxes (low/no expression) from the heatmap are shown on the Brick plots (Fig. 2b, Additional file 1).

We used mass cytometry data acquired from human colorectal tumour and nontumour bowel (NTB) samples as a test dataset to validate the use of Brick plots to identify canonical and novel immune cell populations. This data had previously been assessed using SCAFFoLD [1]. Here we used $\mathrm{x}$-shift in the VorteX application to define cell clusters and generated Brick plots of five out of ten of the major clusters descried by VorteX [7] (Fig. 3).

The Brick plots provided a clear visualization of canonical immune cell populations but also revealed unexpected populations. Markers were grouped into four clusters, indicated by color, by K-means clustering (Fig. 3a). T cell markers were predominantly in pink, resident myeloid cell markers in orange, natural killer (NK) cells in light purple and activated myeloid cell markers in dark purple.

We identified activated CD4 T cells (Fig. 3b), CD8 T cells (Fig. 3c), an NK cell population (Fig. 3d), a resident myeloid population [9] (Fig. 3e), and an additional myeloid population with high expression of markers including CD25 (bottom centre, Fig. 3f). Importantly, co-expression or mutually exclusive expression was consistent with previous reports. By grouping the bricks based on co-expression, it was easy to identify $\mathrm{T}$ cell populations (Fig. 3b, c) by their pink brick predominance versus myeloid cell populations (Fig. 3e, f) with dark purple and orange brick dominance.

The Brick plot in Fig. 3b shows an activated CD4 T cell population. The population has large CD3 and CD4 bricks (centre) indicating a CD4 $\mathrm{T}$ cell population. In addition, 


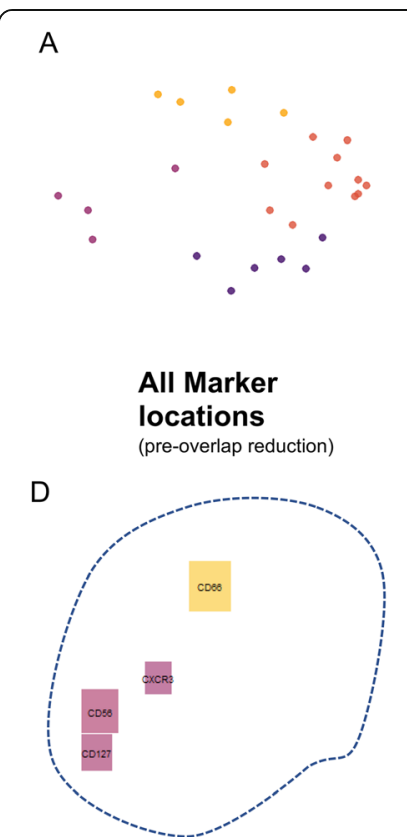

NK cell
B

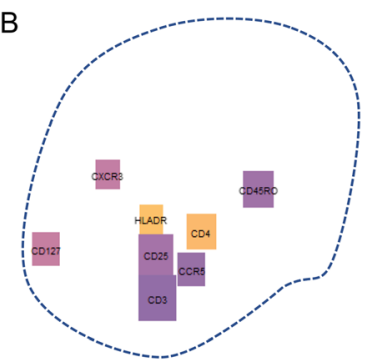

Activated CD4

$T$ cell

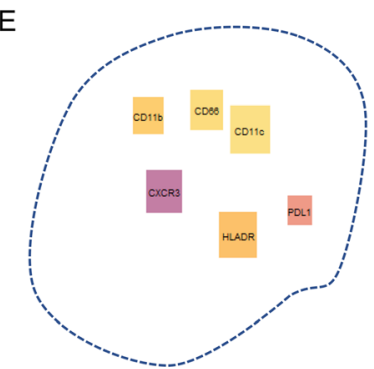

Resident Myeloid

population

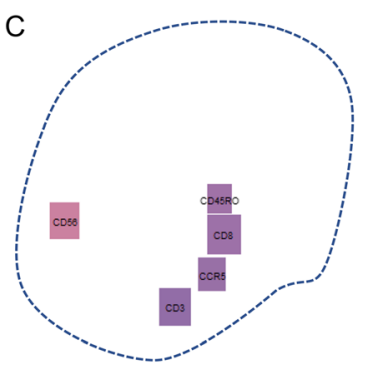

CD8 T cell

F

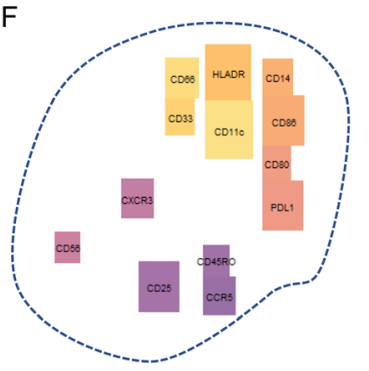

Highly active Myeloid population

G

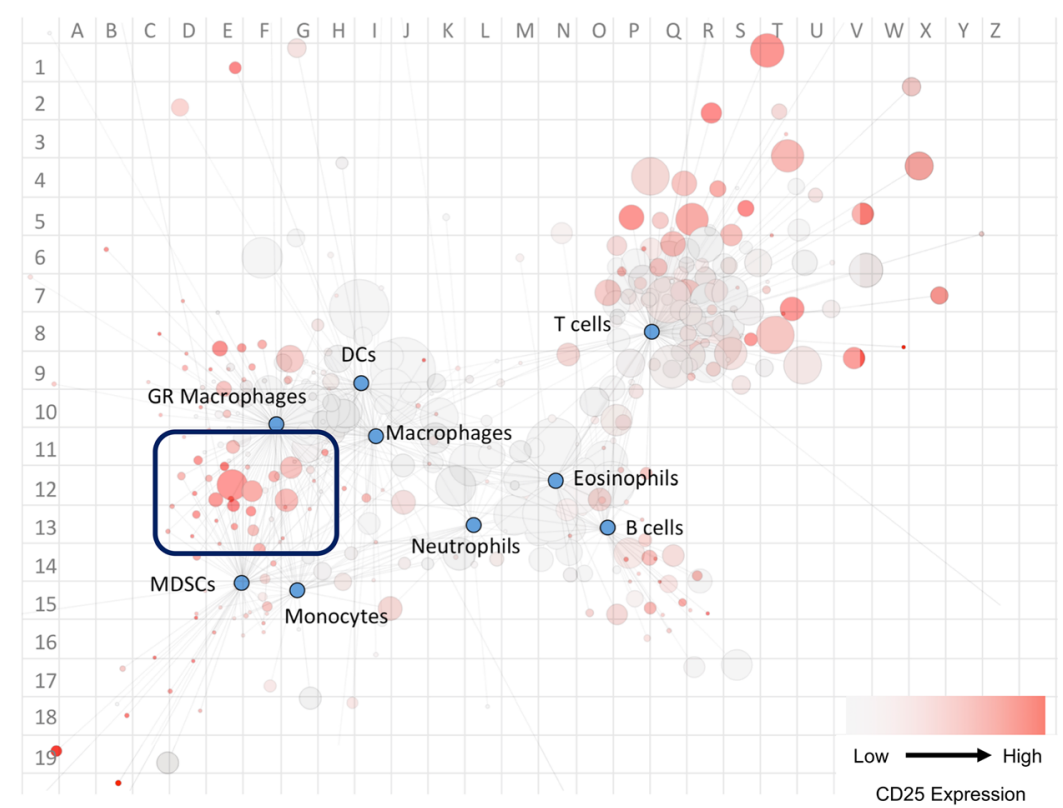

Fig. 3 Brick plots enable visualization of immune cell populations. Data used was acquired by mass cytometry from CRC patient cohort 2 (pooled, Additional file 6). a-f Brick plots generated downstream of xshift clustering in VorteX. (A) all marker locations defined by PCA and coloured by K-means cluster assignation - pink represents markers associated with T cells, orange represents markers associated with resident myeloid cells, light purple represents markers associated with NK cells and dark purple represents markers associated with activated myeloid cells. b-f Brick size indicates relative expression of the labelled marker, colour indicates marker cluster assignation. g SCAFFoLD plot generated using the same dataset, with grid reference overlay added post-analysis. Blue dots indicate predefined landmark nodes, grey (low) to red (high) dots indicate relative CD25 expression. The size of each node indicates relative population size. The purple box outlines the CD25hi myeloid populations depicted in (f) 
this population has large CD45RO (right) and intermediate CD25 (centre) bricks indicating an activated CD4 $\mathrm{T}$ cell phenotype. In comparison, the population depicted in Fig. 3c has a large CD8 brick and intermediate CD3 (centre), corresponding to CD8 T cells. And, while still expressing CD45RO, the relative size of this brick compared to Fig. $3 \mathrm{~b}$ indicates lower expression of this markers in this population. The presence of a small CD56 brick (left) in Fig. 3c is likely the result of the general low CD56 expression within the assessed dataset. Genuine CD56 expression is depicted in the NK cell population in Fig. 3d has a large CD56 brick compared to Fig. 3c.

The list of immunological markers included in this panel was not targeted towards identifying NK cell population. The NK cell population in Fig. 3d had high expression of CD56, CD66 and CD127 - the interpretation of these data suggests a possible NK/ innate lymphoid cell (ILC)3 population [10,11] or even a T cell population [12]. This identification of an NK cell population highlights the capability of Brick plots to correctly reveal populations that were not explicitly targeted without creating noise from a large number of negative markers.

\section{Brick plots provide an intuitive visualization of unpredicted populations}

The presence of a CD25 $5^{\text {hi }}$ macrophage-like population in the tumour tissue was a key finding from the previously unpublished analysis of this dataset in SCAFFoLD (Fig. 3g). Broad lineage-level populations were defined prior to the SCAFFoLD analysis using conventional cytometry approaches such as bivariate gating. These populations formed the "scaffold" of blue nodes in the SCAFFoLD plot. Grey to red nodes represent raw data. The size of a node indicates the relative abundance of that exact phenotype. The proximity of a raw node to a landmark indicates its similarity to the initially defined lineage. In this particular plot, the grey to red axis indicates relative low to high CD25 expression. The purple box at co-ordinates C-G, 11-13 (Fig. 3g) highlights a population of CD25 high cells that are situated closely to myeloid lineages, particularly macrophage subsets - defined by expression of CD64, CD33, CD11b, CD14, HLA-DR at various levels. This population is more clearly described as the Brick plot, Fig. 3f. The large relative size of all dark purple and orange bricks, that represent common myeloid markers, in addition to a large CD25 brick (bottom) immediately reveals a CD25 ${ }^{\mathrm{Hi}}$ myeloid population. This population also expresses markers more commonly associated with $\mathrm{T}$ cell subsets, such as CD45RO and CCR5 (bottom right). However, the population in Fig. 3f does not express CD3, and further, it is possible for these markers to be expressed in myeloid populations [13-15]. We also verified the existence of this CD25 myeloid population by manual gating (Additional file 3). To ascertain the same information displayed in the Brick plot using SCAFFoLD, the researcher would have to cycle through every available marker in the same manner as CD25 is portrayed in Fig. 3g, and manually list marker expression.

\section{Brick plots are also useful for low parameter flow cytometry data}

The increase in available parameters was the main justification for the development of Brick plots. However, low parameter analyses may also benefit from alternative visualization methods to define population phenotypes. In our previous work, we used flow cytometry to define macrophage populations in tumour and non-tumour bowel 
from people with colorectal cancer [9]. Considerable effort was made to design a gating strategy using conventional bivariate gating methods in order to define these macrophage populations. We have subsequently used cluster analyses and Brick plots to re-assess these populations (Fig. 4a, b). Our results mirrored our initial findings [9]; we defined a gut resident macrophage population (Fig. 4a) and a more activated macrophage population (Fig. 4b). These Brick plots thus describe the same phenotypes as conventional methods, but in a simpler format, and via a more streamlined pipeline.

Many clinical trials are interested in immune cell populations as blood based biomarkers. We used Brick plots to assess a mass cytometry dataset generated from a recent pomalidomide/dexamethasone versus pomalidomide alone treatment trial in patients with multiple myeloma (Fig. 4c-e). All samples analyzed were from treated patients. The panel was targeted towards the identification of all PBMC immune subsets. Using Brick plots, we readily identified T cell populations (Fig. 4c), multiple NK cell populations (Fig. 4d and e), and B cell and myeloid derived subsets (data not shown).

\section{Discussion}

We aimed to streamline the analysis process for mass cytometry data analysis. Current approaches are excellent at reducing the high parameter data to a comprehensible level. However, understanding the phenotypes of each of the populations defined using these approaches is still a laborious process. Brick plots represent a new approach to visualizing the phenotype of an immune cell population defined by cluster analyses. Brick plots

A

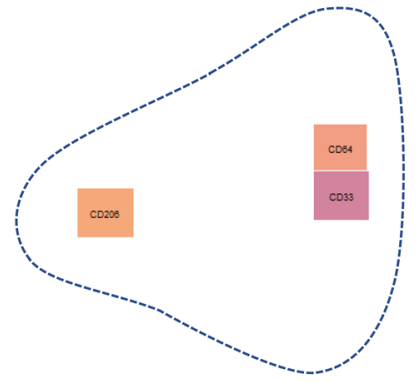

Anti-inflammatory Gut

Resident Macrophage
B

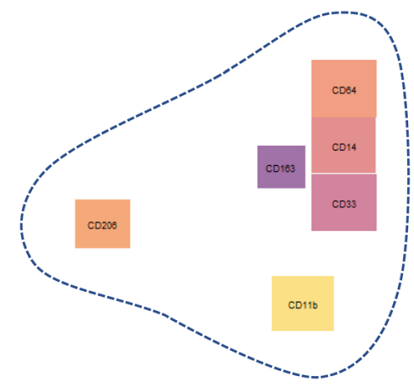

Activated Macrophage

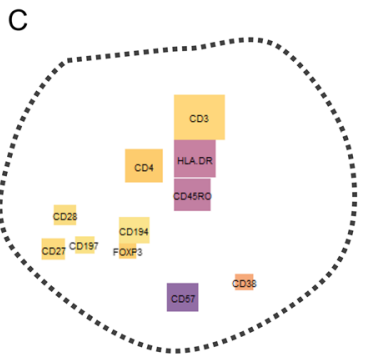

Activated CD4 T cell

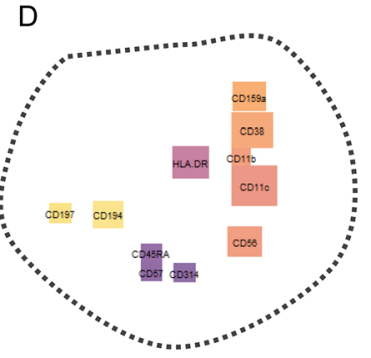

NK Cell

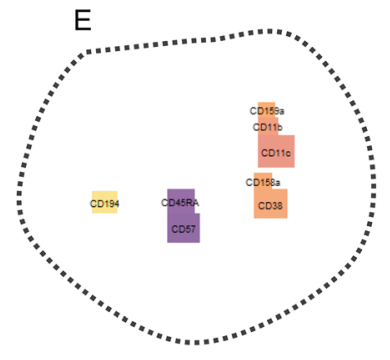

NK Cell

Fig. 4 Brick plots can be used to visualise data acquired by flow cytometry and data from clinical trials. Brick plots generated downstream of $x$-shift clustering in VORTEX. Brick size indicates relative expression of the labelled marker, colour indicates cluster assignation. $\mathbf{a}-\mathbf{b}$ Data used was acquired by flow cytometry from CRC patient cohort 3 (pooled, Additional file 7). c-e Data used was acquired by mass cytometry from MM patient cohort 4 (pooled, Additional file 8) 
are based on a two-step process to produce an intuitive visualization of cluster phenotype using a two-dimensional barcode of bricks representing marker expression.

t-Distributed Stochastic Neighbor Embedding (tSNE) and Spanning-tree Progression Analysis of Density-normalized Events (SPADE) (or other MST variants) are commonly used to analyse mass cytometry data. Korin et al., 2018 [16] described methods for using both tSNE (viSNE) and SPADE to analyse immune cell populations in the brain. Using this approach, the authors precisely defined a broad range of immune cells in the brain and could delineate them from non-immune cell populations based on CD45 expression. The authors state that the SPADE plot generated is an accumulation of several individually assessed SPADE plots which highlights the convoluted nature of defining population phenotypes using this approach. The use of Brick plots as a final step to their existing analysis would greatly streamline population phenotyping and provide an alternate visualization method.

Horowitz et al, 2013 [17] used mass cytometry to assess human NK cell receptor repertoire and revealed a high degree of diversity within NK cell populations in human peripheral blood. Their analysis approach included both SPADE plots and heatmaps. The authors also implemented a one-dimensional barcode system for defining NK cell subsets based on marker expression. Brick plots could have been used in this regard to provide a more informative phenotypic description of the individual NK cell populations. Horowitz et al, 2013 [17] also highlighted the complexity of presenting and describing phenotypes using multiple SPADE plots - an issue that arises with any MST or force-directed layout. An individual plot must be presented for each assessed marker, whereas all markers are displayed on a single plot with Brick plots. The addition of Brick plots to some analyses would allow clearer representation of key findings by reducing complexity due to noise.

Heatmaps are the universal tool, across many scientific disciplines, for displaying complex descriptive data. Heatmaps use colored boxes in a grid to represent relative high or low values for a given variable (column) in a given data point (row). In biology, this representation usually equates to a compact readout showing relative expression of every assessed variable (gene, protein or other) in every biological sample. For the remainder of this discussion, heatmaps will be referred to in the context of biological use. Broad patterns can be easily identified in a heatmap, but subtle population changes are not immediately recognisable. In this regard Brick plots provide a complementary alternative; providing information on the phenotype of a specific cell type, rather than a summary of all available data. In a Brick plot, in contrast to a heatmap, only the relevant markers are displayed, thus reducing noise and aiding in immediate interpretability. Heatmaps often include hierarchical clustering of variables and/or samples. This clustering allows for broad pattern recognition and also gives an indication of marker co-expression. Brick plots use K-means clustering and marker location designation by PCA to display marker co-expression. Bottcher et al used mass cytometry to study human microglial and mononuclear cell subsets [18]. The data presented in a heatmap clearly identifies clusters of cells based on groups of phenotypic markers and separates the sources of the cell types. Brick plots, used for these data, could more easily compare the $\mathrm{T}$ cell populations from peripheral blood mononuclear cells (PBMCs) versus cerebrospinal fluid (CSF) to identify differences in expression of common markers (such as CD3 (higher on PBMCs)) and differentially expressed markers (such as PDL-1(only on CSF cells) and CD45 (only on PBMCs)). 
Mass cytometry analysis was the specific focus for the development of Brick plots. We wanted to develop a tool that was intuitive to immunologists without a strong bioinformatic background and thereby increase the accessibility of high parameter analyses. Moreover, it was important that Brick plots could be used downstream of any of the existing approaches to analysing mass cytometry data $[1,4,6,7]$. Brick plots are not meant as a replacement for these techniques, but as an addition, to improve interpretability and provide an alternate method of presenting data. Brick plots can be generated from any dataset that produces a table of samples (clusters, populations) against markers (protein, gene or other). We produced Brick plots from mass cytometry data generated from a clinical trial of multiple myeloma treatment options. We showed that Brick plots can be used to make complex immunological populations more readily understandable by clinicians, who require rapid data interpretation to generate useful correlations with treatment efficacy. A shift towards a standard of high parameter analyses in clinical trials could be made easier by visualization approaches like Brick plots.

While we provide illustration of the use of Brick plots for display of both mass cytometry and flow cytometry data, this approach would be just as valid for describing gene expression data. For gene data, the localization of commonly co-expressed (or not co-expressed) markers within the Brick plot could provide a useful visualization to determine patterns of expression in different samples or treatment groups. With an even broader scope, Brick plots could be used to define different microbial communities. For this, each brick would represent a different species or sub-species and the size of each brick the relative abundance of that species. For studies of the microbiota, this could provide a useful tool for describing changes in different disease states, or after dietary alterations. Vich Vila et al., 2018 [19] used shotgun meta-genomic sequencing to define differences in the microbiota of patients with various forms of inflammatory bowel diseases (IBD). They produced a branching visualization to describe differences in subspecies abundance between three groups of patients. Brick plots could instead have been used to provide a concise summary of the different microbial compositions; each brick would represent a species and its size the relative abundance. Changes in brick size between the three plots would highlight differences. Moreover, co-localization of species within the Brick plot could help to identify patterns of co-colonization.

Brick plots are more immediately interpretable than other available visualization techniques largely due to the removal of irrelevant markers and the grouping of commonly co-expressed markers. A single Brick plot is generated for each cluster. Brick plots were designed in this manner to expedite the phenotypic description of a given cluster. However, in some instances, understanding broad changes with respect to a given marker across all samples is a more important output. Brick plots are not well suited to this type of analysis - existing approaches, including those described in Fig. 1, would be more appropriate. Spitzer et al, 2015 [1] used SCAFFoLD to describe changes in PBMC immune cell composition within normal circadian rhythm. The authors were primarily interested in broad changes to the immune "landscape" as a whole, making specific population phenotyping unnecessary. A further limitation to Brick plots is the issue of excessive brick overlap when too many markers correlate to a high degree, and the need to manually move brick labels for clarity. This reduces interpretability considerably and so methods may need to be implemented within Brick plots to detect and resolve these overlaps. 


\section{Conclusion}

Brick plots represent a new and novel approach to visualizing cluster phenotypes generated from highly parametric datasets. This approach was developed to streamline the process of describing populations defined by existing analysis approaches. While Brick plots were designed for use with mass cytometry data, they are also well suited to other data types including those outside of immunology. Most importantly, Brick plots provide a more intuitive approach to defining population phenotypes that should prove accessible to users with a wider range of skillsets than currently available methods.

\section{Methods}

\section{Patients}

Tissue samples for Cohorts 1, 2 and 3 (Additional files 5, 6 and 7) were obtained from patients undergoing elective surgery for colorectal cancer (CRC) at Dunedin Hospital. The study was approved by the Health and Disability Ethics Committee (\#14/NTA/33) and all patients gave written informed consent prior to inclusion in the study in accordance with the Treaty of Helsinki. Specimens were dissected by a pathologist.

For Cohort 4 (Additional file 8), peripheral blood (PB, $10 \mathrm{~mL}$ ) from multiple myeloma (MM) patients was obtained following written informed consent as per the Alfred Hospital Human Ethics Committee-approved protocol. ALLG MM14, Universal Trial Number U1111-1126-2829 was approved by the Alfred Office of Ethics and Research Governance. (\#546/12) Trial public title: A prospective randomized Phase II study of single agent pomalidomide maintenance versus combination pomalidomide and low dose dexamethasone maintenance following induction with the combination of pomalidomide and low dose dexamethasone in patients with relapsed and refractory myeloma previously treated with lenalidomide.

\section{Brick plots code}

All code for Brick plots was written in $\mathrm{R}$ using the $\mathrm{R}$ Studio IDE. Fully commented code and instructions for new users can be found at (https://github.com/NortonS1/ BrickPlots). Test files have been provided.

\section{VorteX and SCAFFoLD cluster analyses}

Analyses were performed using the validated SCAFFoLD [1] and VorteX [7] packages as per the developers' instructions. Pre-processing of data was performed in FlowJo (version X.0.7, Tree Star), and some basic reorganization of data was performed in R.

Specific settings in both VorteX and SCAFFoLD were dependent on the input samples from each cohort. Default settings were used for all options other than marker selection.

\section{Minimum spanning tree and Heatmap analyses}

Heatmaps were produced using the standard heatmap package included in base $\mathrm{R}$. Minimum Spanning Trees were produced in the VorteX package using the MST visualisation to retain the same cluster identities. 


\section{Tissue and PB immune cell isolation}

CRC tissue samples were processed as described [20, 21]. Briefly, samples were collected and digested prior to mechanical dissociation. Samples were filtered to remove additional tissue debris. For mass cytometry, samples were frozen in liquid nitrogen.

PB samples were subjected to Ficoll isolation of mononuclear cells. Red blood cells were removed by red blood cell (RBC) lysis buffer (Sigma Aldrich, St. Louis, MO). Cells were pelleted then frozen in liquid nitrogen in 10\% dimethylsulfoxide (DMSO; Sigma Aldrich)/fetal calf serum (FCS;Life Technologies, Carlsbad, CA).

\section{Mass cytometry and flow cytometry acquisition}

Cryopresereved cells were transported to the mass cytometry facility at the Ramaciotti Centre for Human Systems Biology, University of Sydney, Australia, for acquisition on a Helios upgraded CyTOF2 (Fluidigm, South San Francisco, CA, USA). For flow cytometry, samples were prepared as for mass cytometry, but instead acquired immediately on an LSR-FORTESSA (Becton Dickinson, Franklin Lakes, NJ) at the University of Otago flow cytometry facility, Dunedin, New Zealand.

For the CRC cohorts, mass cytometry samples and flow cytometry samples were incubated with antibodies and processed as previously described [20, 21]. For mass cytometry, all reagents were provided by the Ramaciotti Centre for Human Systems Biology, at the University of Sydney. Samples were incubated with cisplatin (to identify viability), blocked with Fc Receptor antibody and incubated with a metal tagged antibody master mix (Additional file 5).

For the MM cohort, $24 \mathrm{~h}$ prior to incubation with antibodies, cells were thawed and incubated overnight at $37^{\circ} \mathrm{C}$ in complete RPMI 1640 media (Thermofisher) consisting of $10 \%$ FCS. Cells were washed in CyTOFACS buffer (PBS (Life Technologies), 0.1\%BSA (Sigma Aldrich), 2 mM EDTA (Sigma Aldrich),0.05\% sodium azide (Sigma Aldrich)) and barcoded using Cell-ID 20-Plex Pd Barcoding Kit (Fluidigm) as per manufacturer's protocol. Post bar coding, cells were incubated with surface antibodies followed by intracellular antibodies using Permeabilization Buffer (eBioscience, San Diego, CA). Dead cells were identified by cisplatin. Samples were resuspended in mQwater and filtered through a $70 \mu \mathrm{m}$ mesh before analysis.

For flow cytometry, samples were incubated with a live/dead viability dye and incubated with fluorescently labelled antibodies [21] (Additional file 6).

\section{Supplementary information}

Supplementary information accompanies this paper at https://doi.org/10.1186/s12859-020-3469-y.

Additional file 1. Heatmap. MST and Brick plot depicting cluster phenotypes for Cohort 1. T cell populations generated from colorectal cancer patient Cohort 1 tissue samples (pooled) acquired by mass cytometry (Additional file 5). (A) Relative expression of markers across all clusters indicated from red (low) to green (high); cluster 20,311 highlighted for comparison in (B-C). From Fig. 2b, Cluster 20,313 is cluster A; Cluster 20,315 is cluster B. (B) MST plot of the T cell populations shown in A, colored for FOXP3; cluster 20,311 highlighted; one of 34 possible SPADE plots that could be generated (see Additional file 2). (C). Brick plot of cluster 20,311, demonstrating expression of all phenotypic markers.

Additional file 2. MST plots of all clusters. T cell populations generated from colorectal cancer patient Cohort 1 tissue samples (pooled) acquired by mass cytometry (Additional file 5). All clusters shown in Additional file 1 shown as MST plots.

Additional file 3. Manually gated $\mathrm{CD} 25^{+}$myeloid cells. Data used was acquired by mass cytometry from CRC patient cohort 2 (pooled, Additional file 6). (Top) pooled cells from non-tumour bowel tissue, (Bottom) pooled cells from CRC tumour tissue. Cells shown were pre-gated on cisplatin ${ }^{-}$, DNA ${ }^{+}$. Cells were gated on CD3 ${ }^{-}$, followed by $\mathrm{CD}_{64}{ }^{+}$to define a broad myeloid population. CD25 expression was assessed on these myeloid cells. 
Additional file 4. Cut off values. A histogram of CD45RO as an example of a cut off value. The cut off value in this instance is similar to a conventional gating strategy used for traditional flow cytometry analysis. This means that the zero value is decided based on the biological data, which will vary for each experiment and by each user depending on the research question asked. This process, while guided by controls, is somewhat subjective and requires learned expertise and a knowledge of the specific dataset. Setting this threshold for the Brick plots package usually requires assessing the raw data to determine the maximum background signal.

Additional file 5. Mass Cytometry Antibody Panel 1. Mass cytometry panel to assess colorectal cancer tissue samples (Cohort 1; $n=20$ ).

Additional file 6. Mass Cytometry Antibody Panel 2. Mass cytometry panel to assess colorectal cancer tissue samples (Cohort 2; $n=3$ ).

Additional file 7. Flow Cytometry Antibody Panel 1. Mass cytometry panel to assess colorectal cancer tissue samples (Cohort 3; $n=11$ ).

Additional file 8. Mass Cytometry Antibody Panel 3. Mass cytometry panel to assess blood samples from multiple myeloma patients (Cohort 4; $n=161$ ).

\section{Abbreviations}

CD-: Cluster of differentiation; CRC: Colorectal cancer; CTLA-4: Cytotoxic T lymphocyte antigen - 4; CyTOF: Cytometry by time of flight; FCS: Foetal calf serum; IBD: Inflammatory bowel diseases; ILC: Innate lymphoid cell; MM: Multiple myeloma; MST: Minimum spanning tree; NK: Natural killer; NTB: Non-tumour bowel; PBMC: Peripheral blood mononuclear cells; PCA: Principal component analysis; RBC: Red blood cell; SCAFFoLD: Single-Cell Analysis by Fixed Force- and Landmark-Directed; SPADE: Spanning-tree Progression Analysis of Density-normalised Events; Tbet: T-box protein expressed in T cells; tSNE: T-Distributed Stochastic Neighbour Embedding; viSNE: T-Distributed Stochastic Neighbour Embedding -based visualisation

\section{Acknowledgements}

We thank all of the patients and donors who contributed to this study, and to John McCall and Fran Munro who facilitated the CRC studies. We thank Justin Tirados for review of the manuscript.

\section{Authors' contributions}

SN performed analysis and conceived the idea, SN, JL, RK, TK and AS provided data. HM and BF provided analysis support. SN and RK wrote the manuscript with input and analysis from HM and BF. All authors have read and approved the final manuscript.

\section{Funding}

This research was funded by the Cancer Research Trust New Zealand, Lottery Health Research New Zealand (CRC studies) and the Australasia Leukemia and Lymphoma Group (MM study). RK was supported by the New Zealand Society for Oncology-Roche Translational Research Fellowship. JL was supported by a University of Otago MSc scholarship. HM was previously supported by an Australian National Health and Medical Research Council Early Career Fellowship (GNT1037298) and is currently supported by the International Society for the Advancement of Cytometry (ISAC) Marylou Ingram Scholars program. The funding bodies played no role in the design of the study or collection, analysis, or interpretation of data and in writing the manuscript.

\section{Availability of data and materials}

The datasets generated and/or analysed during the current study are not publicly available due to patient confidentiality but are available from the corresponding author on reasonable request.

\section{Ethics approval and consent to participate}

For Cohorts 1,2 and 3, the study was approved by the Health and Disability Ethics Committee (\#14/NTA/33) and all patients gave written informed consent prior to inclusion in the study in accordance with the Treaty of Helsinki. For Cohort 4, peripheral blood (PB, $10 \mathrm{~mL}$ ) from multiple myeloma (MM) patients was obtained following written informed consent as per the Alfred Hospital Human Ethics Committee-approved protocol. ALLG MM14, Universal Trial Number U1111-1126-2829 was approved by the Alfred Office of Ethics and Research Governance. (\#546/12) Trial public title: A prospective randomized Phase II study of single agent pomalidomide maintenance versus combination pomalidomide and low dose dexamethasone maintenance following induction with the combination of pomalidomide and low dose dexamethasone in patients with relapsed and refractory myeloma previously treated with lenalidomide.

\section{Consent for publication}

Not applicable.

\section{Competing interests}

The authors declare that they have no competing interests.

\section{Author details}

${ }^{1}$ Department of Microbiology and Immunology, University of Otago, Dunedin, New Zealand. ${ }^{2}$ Myeloma Research Group, Australian Centre for Blood Diseases, Alfred Hospital-Monash University, Melbourne, VIC, Australia. ${ }^{3}$ Malignant Hematology and Stem Cell Transplantation, Alfred Hospital, Melbourne, VIC, Australia. ${ }^{4}$ Ramaciotti Facility for Human Systems Biology, The University of Sydney and Centenary Institute, Sydney, Australia. ${ }^{5}$ Discipline of Pathology, School of Medical Sciences, Faculty of Medicine and Health, The University of Sydney, Australia; Charles Perkins Centre, University of Sydney, Sydney, Australia. 
Received: 6 November 2019 Accepted: 24 March 2020

Published online: 15 April 2020

\section{References}

1. Spitzer MH, Gherardini PF, Fragiadakis GK, Bhattacharya N, Yuan RT, Hotson AN, et al. An interactive reference framework for modeling a dynamic immune system. Science (New York, NY). 2015;349(6244):1259425.

2. Papageorgiou L, Eleni P, Raftopoulou S, Mantaiou M, Megalooikonomou V, Vlachakis D. Genomic big data hitting the storage bottleneck. EMBnetjournal. 2018;24:e910.

3. Bendall SC, Simonds EF, Qiu P, El-ad DA, Krutzik PO, Finck R, et al. Single-cell mass cytometry of differential immune and drug responses across a human hematopoietic continuum. Science. 2011;332(6030):687-96.

4. Bruggner RV, Bodenmiller B, Dill DL, Tibshirani RJ, Nolan GP. Automated identification of stratifying signatures in cellular subpopulations. Proc Natl Acad Sci. 2014;111(26):E2770-E7.

5. Irish JM, Doxie DB. High-dimensional single-cell cancer biology, High-Dimensional Single Cell Analysis. Berlin: Springer; 2014. p. 1-21.

6. Qiu P, Simonds EF, Bendall SC, Gibbs KD Jr, Bruggner RV, Linderman MD, et al. Extracting a cellular hierarchy from highdimensional cytometry data with SPADE. Nat Biotechnol. 2011;29:886.

7. Samusik N, Good Z, Spitzer MH, Davis KL, Nolan GP. Automated mapping of phenotype space with single-cell data. Nat Methods. 2016;13(6):493.

8. Spitzer MH, Nolan GP. Mass Cytometry: single cells, Many Features. Cell. 2016;165(4):780-91.

9. Norton SE, Dunn ET, McCall $J$, Munro F, Kemp RA. Gut macrophage phenotype is dependent on the tumor microenvironment in colorectal cancer. Clin Transl Immunol. 2016;5(4):e76.

10. Cupedo T, Crellin NK, Papazian N, Rombouts EJ, Weijer K, Grogan JL, et al. Human fetal lymphoid tissue-inducer cells are interleukin 17-producing precursors to RORC+ CD127+ natural killer-like cells. Nat Immunol. 2009;10(1):66-74.

11. Mahapatra S, Mace EM, Minard CG, Forbes LR, Vargas-Hernandez A, Duryea TK, et al. High-resolution phenotyping identifies NK cell subsets that distinguish healthy children from adults. PLoS One. 2017;12(8):e0181134.

12. Stewart CA, Walzer T, Robbins SH, Malissen B, Vivier E, Prinz I. Germ-line and rearranged Tcrd transcription distinguish bona fide NK cells and NK-like gammadelta T cells. Eur J Immunol. 2007;37(6):1442-52.

13. Pilling D, Fan T, Huang D, Kaul B, Gomer RH. Identification of markers that distinguish monocyte-derived fibrocytes from monocytes, macrophages, and fibroblasts. PLoS One. 2009;4(10):e7475.

14. Shaheen ZR, Christmann BS, Stafford JD, Moran JM, Buller RML, Corbett JA. CCR5 is a required signaling receptor for macrophage expression of inflammatory genes in response to viral double-stranded RNA. Am J Physiol Regul Integr Comp Physiol. 2019;316(5):R525-R34.

15. Gordon SR, Maute RL, Dulken BW, Hutter G, George BM, McCracken MN, et al. PD-1 expression by tumour-associated macrophages inhibits phagocytosis and tumour immunity. Nature. 2017:545(7655):495-9.

16. Korin B, Dubovik T, Rolls A. Mass cytometry analysis of immune cells in the brain. Nat Protoc. 2018;13:377.

17. Horowitz A, Strauss-Albee DM, Leipold M, Kubo J, Nemat-Gorgani N, Dogan OC, et al. Genetic and Environmental Determinants of Human NK Cell Diversity Revealed by Mass Cytometry. Sci Transl Med. 2013:5(208):208ra145.

18. Bottcher C, Schlickeiser S, Sneeboer MAM, Kunkel D, Knop A, Paza E, et al. Human microglia regional heterogeneity and phenotypes determined by multiplexed single-cell mass cytometry. Nat Neurosci. 2019;22(1):78-90.

19. Vich Vila A, Imhann F, Collij V, Jankipersadsing SA, Gurry T, Mujagic Z, et al. Gut microbiota composition and functional changes in inflammatory bowel disease and irritable bowel syndrome. Sci Transl Med. 2018;10(472):eaap8914.

20. Norton SE, Ward-Hartstonge KA, McCall JL, Leman JK, Taylor ES, Munro F, et al. High-dimensional mass cytometric analysis reveals an increase in effector regulatory $T$ cells as a distinguishing feature of colorectal tumors. J Immunol. 2019:202(6):1871-84.

21. Taylor ES, McCall JL, Shen S, Girardin A, Munro FM, Black MA, et al. Prognostic roles for IL-2-producing and CD69+ T cell subsets in colorectal cancer patients. Int J Cancer. 2018;143(8):2008-16.

\section{Publisher's Note}

Springer Nature remains neutral with regard to jurisdictional claims in published maps and institutional affiliations.

\section{Ready to submit your research? Choose BMC and benefit from:}

- fast, convenient online submission

- thorough peer review by experienced researchers in your field

- rapid publication on acceptance

- support for research data, including large and complex data types

- gold Open Access which fosters wider collaboration and increased citations

- maximum visibility for your research: over $100 \mathrm{M}$ website views per year

At $\mathrm{BMC}$, research is always in progress.

Learn more biomedcentral.com/submissions 